\title{
Fault Tolerant Boolean Satisfiability
}

\author{
Amitabha Roy \\ Computer Science Department, \\ Boston College, Chestnut Hill, MA 0246\%.
}

AROY@CS.BC.EDU

\begin{abstract}
A $\delta$-model is a satisfying assignment of a Boolean formula for which any small alteration, such as a single bit flip, can be repaired by flips to some small number of other bits, yielding a new satisfying assignment. These satisfying assignments represent robust solutions to optimization problems (e.g., scheduling) where it is possible to recover from unforeseen events (e.g., a resource becoming unavailable). The concept of $\delta$-models was introduced by Ginsberg, Parkes, and Roy (1998), where it was proved that finding $\delta$-models for general Boolean formulas is NP-complete. In this paper, we extend that result by studying the complexity of finding $\delta$-models for classes of Boolean formulas which are known to have polynomial time satisfiability solvers. In particular, we examine 2-SAT, Horn-SAT, AffineSAT, dual-Horn-SAT, 0-valid and 1-valid SAT. We see a wide variation in the complexity of finding $\delta$-models, e.g., while 2-SAT and Affine-SAT have polynomial time tests for $\delta$-models, testing whether a Horn-SAT formula has one is NP-complete.
\end{abstract}

\section{Introduction}

An important problem in the artificial intelligence community concerns the allocation of resources at or near the minimal cost. An optimal solution to such a problem might be rendered infeasible due to some unforeseen event (for example, a resource becoming unavailable or a task exceeding its allocated deadline). Hence, the motivation is to search for optimal solutions which are immune from such events. In this paper, we consider the complexity of finding such "robust" solutions, where we only allow for a fixed small number of bad events, with the added condition that such bad events can be rectified by making a small change to the solution. These solutions, which we call $\delta$-models, were introduced by Ginsberg et al. (1998), and further explored in Bailleux and Marquis (1999). This approach to fault tolerance has been extended to constraint-satisfaction problems (CSPs) (Hebrard, Hnich, \& Walsh, 2004b, 2004a) and to applications in combinatorial auctions (Holland \& O'Sullivan, 2004). Hoos and O'Neill (2000) consider this approach to robustness in the framework of dynamic satisfiability (which they call DynSAT) where the goal is to be able to revise optimal solutions under a constantly changing input problem.

We extend the initial complexity results in Ginsberg et al. (1998) by looking at the theoretical complexity of tractable instances of satisfiability (SAT) identified by Schaefer's dichotomy theorem (Schaefer, 1978). The dichotomy theorem proves that the polynomial time solvable instances of SAT are 2-SAT, Horn-SAT, dual-Horn-SAT, Affine-SAT, 0-valid SAT and 1-valid SAT and any other form is NP-complete. Our goal is to study the complexity of finding $\delta$-models for the tractable problems identified by the dichotomy theorem. We show a wide variation in complexity by type (2-SAT vs Horn-SAT) and by parameter (the number of repairs allowed for each break). 
Formally, a $\delta$-model of a Boolean formula, called supermodels by Ginsberg et al. (1998), is a satisfying assignment (satisfying assignments are usually called models) such that if any bit of the assignment is flipped (from 0 to 1 or vice versa), one of the following conditions hold:

(i) either the new assignment is a model or

(ii) there is at least one other bit that can be flipped to obtain another model.

Flipping a bit of a $\delta$-model is called a break, corresponding to a "bad" event. The bit that is flipped to get another satisfying assignment is a repair (we allow that some breaks may not need a repair). We also study a generalization of the concept: $\delta(r, s)$-models are satisfying assignments for which breaks to every set of up to $r$ bits need up to $s$ repairs (to avoid trivialities, we require that the repair bits are different from the break bits).

We let $\delta$-SAT refer to the decision question as to whether an input Boolean formula has a $\delta$-model. When we restrict the form of the input Boolean formula, we refer to the corresponding decision questions as $\delta$-2-SAT, $\delta$-Horn-SAT etc. The higher degree variants of these problems are $\delta(r, s)$-SAT etc. where we consider $r$ and $s$ to be fixed integers. The following problems are proved to be NP-complete:

- $\delta(r, s)$-SAT (Ginsberg et al., 1998), $\delta(1, s)$-2-SAT for $s>1$,

- $\delta(1, s)$-Horn-SAT, $\delta(1, s)$-dual-Horn-SAT,

- $\delta(r, s)$-0-valid-SAT and $\delta(r, s)$-1-valid-SAT.

In contrast, we prove that the following problems are in $\mathrm{P}$ :

- $\delta(1,1)$-2-SAT, $\delta(r, s)$-Affine-SAT.

The definition of $\delta$-models does not require that the new model obtained by repairing a break to a $\delta$-model is itself a $\delta$-model. We define $\delta^{*}$-models to be $\delta$-models such that every break needs at most one repair to obtain another $\delta$-model. Such models represent the greatest degree of fault tolerance that can be achieved for the problem. We refer to the corresponding decision problems as $\delta^{*}$-SAT, $\delta^{*}$-2-SAT etc. We prove that $\delta^{*}$-SAT is in NEXP (non-deterministic exponential time) and is NP-hard, $\delta^{*}-2$-SAT is in P and that $\delta^{*}$-Affine-SAT is in P.

Remark. Since our goal in this paper is to study the problems in Schaefer's tractable class with respect to fault tolerance, our yardstick to measure complexity is membership in P. Hence, we do not concern ourselves with finding the exact running times within P. Optimizing runtimes may well prove important for practical applications (at least in the rare instances when we find polynomial time algorithms).

Organization of the paper: In Section 2, we introduce and define the problem and establish notation. In Section 3, we study the complexity of finding $\delta$-models of general Boolean formulas. In Section 4 , we consider the complexity of finding $\delta$-models for restricted classes of formulas: we consider 2-SAT (Section 4.1), Horn-SAT (Section 4.2), 0-valid-SAT, 1-validSAT (Section 4.3) and Affine-SAT (Section 4.4). We conclude with a section on future work (Section 5). 


\section{Definitions and Notations}

In this section, we establish some of the notation used in the rest of the paper and formally define the problems we wish to study.

A Boolean variable can take on two values - true or false which we write as 1 and 0 respectively. A literal is either a variable $v$ or its negation, denoted by $\neg v$ (a variable is often called a pure literal). A clause is a disjunction ( $\vee$ ) of literals (for example, $v_{1} \vee \neg v_{2} \vee v_{3}$ is a clause). A Boolean formula is a function from some set of Boolean variables $V=$ $\left\{v_{1}, v_{2}, \ldots, v_{n}\right\}$ to $\{0,1\}$. In computational problems, we assume that Boolean formulas are input in a canonical fashion: usually as a conjunction $(\wedge)$ of clauses (in which case, we say that they are in conjunctive normal form $(\mathrm{CNF})$ ).

We consider various forms of CNF formulas. A 2-SAT formula is a Boolean formula in $\mathrm{CNF}$ with at most 2 literals per clause (more generally, a $k$-CNF formula or $k$-SAT formula is a CNF formula with $k$ literals per clause). A Horn-SAT formula is a Boolean formula in CNF where each clause has at most one positive literal (each such clause is called a Horn clause). Equivalently, a Horn clause can be written as an implication $\left(\left(v_{1} \wedge v_{2} \ldots \wedge v_{r}\right) \rightarrow u\right)$ where $u, v_{1}, v_{2}, \ldots, v_{r}$ are pure literals and $r \geq 0$. A dual-Horn-SAT formula is a CNF formula where each clause has at most one negative literal. An Affine-SAT formula is a CNF formula in which each clause is an exclusive-or $(\oplus)$ of its literals or a negation of the exclusive-or of its literals (such a clause is satisfied exactly when an odd number of the literals are set to 1). Equivalently, each clause of an Affine-SAT formula can be written as a linear equation over the finite field $\{0,1\}$ of 2 elements.

An assignment is a function $X: V \rightarrow\{0,1\}$ that assigns a truth value (true or false) to each variable in $V$. Given such an assignment of truth values to $V$, any Boolean formula $\phi$ defined over $V$ also inherits a truth value (we denote this by $\phi(X)$ ), by applying the rules of Boolean logic. A model is an assignment $X$ such that $\phi(X)$ is true. We will often treat an assignment $X$ as an $n$-bit vector where the $i$-th bit, denoted by $X(i), 1 \leq i \leq n$, is the truth value of the variable $v_{i}$. With a slight abuse of notation, we let $X(l)$ denote the value of the literal $l$ under the assignment $X$.

A 0-valid-SAT (resp. 1-valid-SAT) formula is one which is satisfied by an assignment with every variable set to 0 (resp. 1 ).

The propositional satisfiability problem is defined as follows:

Problem (SAT).

Instance: A Boolean formula $\phi$.

Question: Does $\phi$ have a model?

SAT is the canonical example of an NP-complete decision problem (for definitions of the complexity class NP and completeness, see e.g., Garey \& Johnson, 1979; Papadimitriou, 1994). Many computational difficult problems in artificial intelligence have $S A T$ encodings (for example, in planning (Kautz \& Selman, 1992)) and so finding heuristic algorithms for solving $S A T$ is an important research area in artificial intelligence. Polynomial time algorithms are known for SAT when the input instance is either Horn-SAT, dual-Horn-SAT, 2-SAT, Affine-SAT, 0-valid-SAT or 1-valid-SAT. Schaefer (1978) proved that these are the only cases when SAT is solvable in polynomial time, every other case being NP-complete 
(Schaefer's theorem applies to a more general situation called "generalized satisfiability" where the truth value of each clause is determined by a set of constraints specified as a relation).

We now introduce the concept of fault-tolerant models. Given an $n$-bit assignment $X$, the operation $\delta_{i}$ flips the $i$-th bit of $X$ (from a 0 to a 1, or vice versa). The operation produces a new assignment which we denote by $\delta_{i}(X)$. Similarly, if we flip two distinct bits (say bits $i$ and $j$ ), we write the new assignment as $\delta_{i j}(X)$ and more generally, $\delta_{S}(X)$ represents $X$ with the bits in $S$ flipped (where $S$ is some subset of the coordinates $\{1,2, \ldots, n\}$ ).

Definition 2.1. A $\delta$-model of a Boolean formula $\phi$ is a model $X$ of $\phi$ such that for all $i$, $1 \leq i \leq n$, either

(i) the assignment $\delta_{i}(X)$ is a model or

(ii) there is some other bit $j$, where $1 \leq j \leq n$ and $i \neq j$, such that $\delta_{i j}(X)$ is a model.

In other words, a $\delta$-model is a model such that if any bit is flipped (we call this a break), at most one other bit flip is required to produce a new model. The second bit flip is called a repair.

Example 2.1. Let $H(n, k)$ be a Boolean formula defined over $n$ variables $v_{1}, v_{2}, \ldots, v_{n}$, whose models are $n$-bit assignments with exactly $k$ bits set to 1 . For example:

$$
H(n, 1)=\left(\bigvee_{i=1}^{n} v_{i}\right) \wedge \bigwedge_{i=1}^{n}\left\{v_{i} \rightarrow\left(\bigwedge_{\substack{j=1 \\ j \neq i}}^{n} \neg v_{j}\right)\right\}
$$

The first clause specifies that at least one bit of a model is 1 and each successive clause specifies that if the $i$-th bit is 1 , then every other bit is set to 0 where $1 \leq i \leq n$. Each model of $H(n, 1)$ is a $\delta$-model: any break to a 0 -bit has a unique repair (the bit set to 1 ) and a break to the 1-bit has $(n-1)$ possible repairs (any one of the 0-bits).

The following decision problem can be interpreted as the fault-tolerant analogue of SAT:

Problem $(\delta$-SAT).

Instance: A Boolean formula $\phi$.

Question: Does $\phi$ have a $\delta$-model ?

The problem $\delta$-SAT and its variants (when we restrict the form of the input Boolean formula) is the focus of this paper.

We now extend our notion of single repairability to repairability of a sequence of breaks to a model.

Definition 2.2. A $\delta(r, s)$-model of a Boolean formula $\phi$ is a model of $\phi$ such that for every choice of at most $r$ bit flips (the "break" set) of the model, there is a disjoint set of at most $s$ bits (the "repair" set) that can be flipped to obtain another model of $\phi$. 
Remark. (i) We view $r$ and $s$ as fixed constants unless otherwise mentioned. To avoid redundancies, we have required that the repair set is disjoint from the break set. Since we require "at most $s$ bits" for repair, we also allow for the case when no repair or fewer than $s$ repairs are needed.

(ii) Under this definition, $\delta(1,1)$-models are $\delta$-models and we continue to refer to them as $\delta$-models for notational simplicity.

(iii) Similar to the definition of $\delta$-SAT, we can define a decision problem $\delta(r, s)$-SAT which asks whether an input Boolean formula has a $\delta(r, s)$-model.

Example 2.2. Each model of $H(n, k)$ (see, e.g., Example 2.1) is also a $\delta(k, k)$ model when $k \leq n / 2$.

Assumptions: In all our discussions, we will assume that every variable of an input Boolean formula appears in both positive and negative literals and that an input Boolean formula is in clausal form with no variable appearing more than once in a clause (i.e., there is no clause of the form $v_{1} \vee \neg v_{1} \vee v_{2}$ ). We also assume that in any instance of $\delta$-SAT (or its variants), there is no clause which consists of a single literal, since in that case the input formula cannot have a $\delta$-model.

Consider a $\delta$-model $X$ of a Boolean formula and suppose that $Y$ is a model which repairs some break to $X$. Our definition (Definition 2.1) of $\delta$-models does not require that $Y$ itself is a $\delta$-model. If we enforce that every break to $X$ is repaired by some $\delta$-model, then not only is $X$ tolerant to a single break, but so is the repair. We thus can define a degree of fault tolerance. In this setting, models will be fault tolerant of degree 0 . Then, $\delta$-models will be fault-tolerant of degree 1 . More generally, degree- $k$ fault-tolerant models (which we call $\delta^{k}$-models) consist of $\delta^{k-1}$ fault-tolerant models such that every break is repaired by a $\delta^{k-1}$ model. We give the formal definition below.

Definition 2.3. Let $\phi$ be a Boolean formula. We define $\delta^{k}(r, s)$-models inductively: $\delta^{0}(r, s)$ models are models of $\phi$. Then for $k \geq 1, \delta^{k}(r, s)$-models of $\phi$ are $\delta^{k-1}(r, s)$-models $X$ of $\phi$ such that for every break of at most $r$ coordinates of $X$, there is a disjoint set of at most $s$ coordinates of $X$ that can be flipped to get a $\delta^{k-1}(r, s)$-model of $\phi$.

We define the corresponding decision problem $\delta^{k}(r, s)$-SAT, which asks whether an input Boolean formula has a $\delta^{k}(r, s)$-model. Observe also that by definition a $\delta^{k}(r, s)$-model is a $\delta^{i}(r, s)$-model for all $i, 0 \leq i \leq k-1$.

Example 2.3. Let $n \geq 6$ be even and let $\phi$ be the Boolean formula:

$$
\left(v_{1}=v_{2}\right) \wedge\left(v_{3}=v_{4}\right) \wedge \cdots\left(v_{n-1}=v_{n}\right) \wedge\left(\bigvee_{k=0}^{4} H(n, k)\right)
$$

Then the models of $\phi$ are vectors with either 0,2 or 4 variables set to 1 . The variables in $\left\{v_{2 i-1}, v_{2 i}\right\}$ have to have the same truth value (and this forces breaks to have unique repairs). 
We claim that $X=(0,0, \ldots, 0)$ is a $\delta^{2}(1,1)$-model of $\phi$. Any break (without loss of generality, assume it is to coordinate 1 ) is repaired by a flip to coordinate 2 (and vice versa). The new vector $(1,1,0,0, \ldots, 0)$ is itself a $\delta$-model. A break to some other coordinate (say, bit 3 ) has a unique repair (bit 4$)$ to give a model $(1,1,1,1,0, \ldots, 0)$ with 41 's. This model is no longer repairable, since any model has to have at most 41 's, so a break to any coordinate with a 0 (e.g., to bit 5) has no repair.

Let $n \geq 2$ be even. Consider the formula

$$
\left(v_{1}=v_{2}\right) \wedge\left(v_{3}=v_{4}\right) \cdots \wedge\left(v_{n-1}=v_{n}\right)
$$

which has $2^{n / 2}$ models. Observe that each model is a $\delta$-model. So these models are $\delta^{k}(1,1)$ models for every integer $k \geq 0$. We call these models $\delta^{*}(1,1)$-models (as usual, when $r=1$ and $s=1$, we denote $\delta^{*}(r, s)$-models as $\delta^{*}$-models for simplicity).

Definition 2.4. Let $\phi$ be a Boolean formula defined over $n$ Boolean variables. Then a model of $\phi$ which is a $\delta^{k}(r, s)$ model for each $k \geq 0$ is called a $\delta^{*}(r, s)$-model.

Observe that the set of all $\delta^{*}$-models of $\phi$ form a set $M$ of models which satisfies the following properties:

(i) Each vector in $M$ is a $\delta$-model, i.e., a break to a bit needs at most 1 repair.

(ii) When any bit of a vector in $M$ is broken, there is some repair (if such a repair is needed) such that the new vector is also a member of $M$.

We call such sets of $\delta^{*}$-models stable sets of $\phi$. These stable sets have been studied in a combinatorial setting by Luks and Roy (2005).

Remark. The existence of families of models which satisfy conditions (i) and (ii) above may be used to give an alternate definition of $\delta^{*}$-models which is perhaps more natural. However, the notion of degrees of repairability and that $\delta^{*}$-models appear as the limit of these degrees, is not apparent from this definition, hence we use the formulation leading to Definition 2.4.

The corresponding decision problem, named $\delta^{*}$-SAT, asks whether an input Boolean formula has a $\delta^{*}$-model. Note that a "yes" answer to this question implies the existence of not one but a family of such models, in particular, a set $M$ as above.

Complexity Classes: We refer to Papadimitriou (1994) for definitions of basic complexity classes like $\mathrm{P}$ and NP. A language $L$ is said to be in NEXP if there is a non-deterministic Turing machine (NDTM) that decides $L$ in exponential time (exponential in the length of the input). A language $L$ is said to be NP-hard if there is a polynomial time reduction from SAT to $L$. A language is NP-complete if it is in NP and is NP-hard. The complexity class NL (non-deterministic log space), which is contained in $\mathrm{P}$, consists of languages that are accepted by non-deterministic Turing machines using space logarithmic in the size of its input. The complexity classes $\Sigma_{k}^{P}$ are defined as follows: $\Sigma_{1}^{P}$ is NP, $\Sigma_{k}^{P}$ for $k \geq 2$ is the set of languages accepted by a NDTM that has access to an oracle TM for $\Sigma_{k-1}^{P}$. 


\section{Complexity of Finding $\delta$-models}

In this section, we study the computational complexity of finding $\delta$-models for general Boolean formulas.

Theorem 3.1. (Ginsberg et al., 1998) The decision problem $\delta(r, s)$-SAT is NP-complete.

Remark. The proof technique used in Ginsberg et al. (1998) to prove Theorem 3.1 is used to prove other NP-hardness results in this paper, e.g., in Theorem 3.2 and Theorem 4.19.

Theorem 3.2. The decision problem $\delta^{*}-S A T$ is in NEXP and is NP-hard.

Proof. Since an NDTM can guess a stable set of models (which could be of exponential size) and check that it satisfies the required conditions for stability in exponential time, $\delta^{*}$-SAT is in NEXP.

We reduce SAT to $\delta^{*}$-SAT using the same reduction used in the proof of Theorem 3.1 in Ginsberg et al. (1998): given an instance $\phi$ of SAT, a Boolean formula $\phi$ over $n$ variables $v_{1}, v_{2}, \ldots, v_{n}$, we construct an instance of $\delta^{*}$-SAT: the formula $\phi^{\prime}=\phi \vee v_{n+1}$ with $v_{n+1}$ being a new variable (to put $\phi^{\prime}$ in $\mathrm{CNF}$ form, we add the variable $v_{n+1}$ to each clause in the CNF formula $\phi)$.

Suppose $\phi$ has a model $X$. We show that $\phi^{\prime}$ has a $\delta^{*}$-model by showing that it has a stable set of models $M$. Extend $X$ to a model $Y$ of $\phi^{\prime}$ by setting $v_{n+1}=0$. Let $X_{i}=\delta_{i}(X)$ for $1 \leq i \leq n$. Extend each assignment $X_{i}$ to a model $Y_{i}$ of $\phi^{\prime}$ by setting $v_{n+1}=1$. Then let

$$
M=\left\{Y, Y_{1}, Y_{2}, \ldots, Y_{n}\right\} .
$$

We now show that $M$ is a stable set. Suppose some bit $j \neq i$, where $1 \leq j \leq n$ of $Y_{i}$ is broken, then repair by flipping the $i$-th bit (in which case, we get the repaired vector $\left.Y_{j} \in M\right)$. If the $i$-th bit of $Y_{i}$ is broken, the repair is the $(n+1)$-th bit (and vice versa), in which case the repaired vector is $Y$. If instead the $i$-th bit of $Y$ is broken, where $1 \leq i \leq n$, then the repair is the $(n+1)$-th bit (we obtain $Y_{i}$ as the repaired vector in this case). If the $(n+1)$-th bit of $Y$ is broken, we can repair by flipping any of the first $n$ bits. Hence $M$ is a stable set of models and so $\phi^{\prime}$ has a $\delta^{*}$-model (in fact, we have exhibited $n+1$ such models).

Now we show that if $\phi^{\prime}$ has a $\delta^{*}$-model, then $\phi$ has a model. If $\phi^{\prime}$ has a $\delta^{*}$-model, it must have a $\delta^{*}$-model with the $(n+1)$-th coordinate set to 0 . Then the restriction of this assignment to $v_{1}, v_{2}, \ldots, v_{n}$ has to be a model of $\phi$. This completes the reduction from SAT.

Remark. Note that while every $\delta^{*}$-model is a $\delta^{k}$-model for each $k \geq 1$, the NP-hardness of $\delta^{*}$-SAT (Theorem 3.2) does not imply the NP-hardness of $\delta^{k}$-SAT (Theorem 3.3 below). The reduction used in Theorem 3.2 can however be adapted to prove Theorem 3.3.

Theorem 3.3. $\delta^{k}$-SAT is NP-complete, where $k \geq 0$.

Proof. When $k=0$, this is Cook's Theorem (Garey \& Johnson, 1979), so assume that $k \geq 1$. First observe that $\delta^{k}$-SAT is in NP. This is because an NDTM can guess an assignment $X$ and check that it is a $\delta^{k}(1,1)$-model: to check whether $X$ is a $\delta^{k}(1,1)$-model, it suffices to 
consider all possible $n^{k}$ break sets, and check that a repair exists for each break applied in sequence from the break set. Since $k$ is fixed, this can be done in polynomial time.

To prove that $\delta^{k}$-SAT is NP-hard, we use, once again, the proof technique used in Ginsberg et al. (1998) to prove Theorem 3.1. Given an instance $\phi$ of SAT, defined on $n$ variables $v_{1}, v_{2}, \ldots, v_{n}$, we construct $\phi^{\prime}=\phi \vee v_{n+1}$ (and modify $\phi^{\prime}$ to a CNF formula), where $v_{n+1}$ is a newly introduced variable. The argument used in Theorem 3.2 can now be used to prove that $\phi$ is satisfiable iff $\phi^{\prime}$ has a $\delta^{k}$-model. In particular, we construct a stable set of models $M$ for $\phi^{\prime}$ from a single model of $\phi$. Since a $\delta^{*}$-model is a $\delta^{k}$-model, this proves that if $\phi$ is satisfiable, then $\phi^{\prime}$ has a $\delta^{*}$-model. The other direction also follows: if $\phi^{\prime}$ has a $\delta^{k}$-model then it has a model with $v_{n+1}$ set to 0 . The restriction of that model to $v_{1}, \ldots, v_{n}$ is a model of $\phi$.

\section{Finding $\delta$-models for Restricted Boolean Formulas}

In this section, we consider the complexity of $\delta(r, s)$-SAT for restricted classes of SAT formulas which are known to have polynomial-time algorithms for satisfiability: 2-SAT, Horn-SAT, dual-Horn-SAT, 0-valid SAT, 1-valid SAT and Affine-SAT. We observe that these problems have different complexity of testing fault tolerance. For example, 2-SAT and Affine-SAT have polynomial time tests for the existence of $\delta$-models (see Section 4.1 and 4.4) whereas the same problem is NP-complete for Horn-SAT (Section 4.2).

\subsection{Finding $\delta$-models for 2-SAT}

We now prove that finding $\delta$-models for 2-SAT formulas is in polynomial time. We give two independent proofs: the first proof (Section 4.1.1) exploits the structure of the formula and the second proof (suggested by a referee) uses CSP (constraint satisfaction problem) techniques (Section 4.1.2). In contrast, we show that finding $\delta(1, s)$-models for 2-SAT formulas is NP-complete for $s \geq 2$ (Section 4.1.3). However, we also show that finding $\delta^{*}$-models for 2-SAT formulas is in polynomial time (Section 4.1.4).

\subsubsection{Polynomial time Algorithm FOR $\delta(1,1)$-2-SAT}

Notation: Let $\phi$ be an instance of 2-SAT. Following the notation in Papadimitriou (1994), we define the directed graph $G(\phi)=(V, E)$ as follows: the vertices of the graph are the literals of $\phi$ and for each clause $l_{i} \rightarrow l_{j}$ (where $l_{i}, l_{j}$ are literals), there are two directed edges $\left(l_{i}, l_{j}\right)$ and $\left(\neg l_{j}, \neg l_{i}\right)$ in $E$. A path in $G(\phi)$ is an ordered sequence of vertices $\left(l_{1}, l_{2}, \ldots, l_{r}\right)$ where $\left(l_{i}, l_{i+1}\right) \in E$ for $1 \leq i \leq r-1$. We define a simple path in $G(\phi)$ to be a path $\left(l_{1}, l_{2}, \ldots, l_{r}\right)$ where the literals $l_{i}$ involve distinct variables, i.e., $l_{i} \neq l_{j}$ and $l_{i} \neq \neg l_{j}$ for all $i \neq j$, where $1 \leq i, j \leq r$. A simple cycle of $G(\phi)$ is a simple path where we allow the start and end vertices to be identical. A source vertex (resp. a sink vertex) in $G(\phi)$ is a vertex with in-degree (resp. out-degree) 0 . A vertex $l$ in $G(\phi)$ is said to be a $k$-ancestor (resp. $k$-descendant) if there exists a simple path $\left(l, l_{1}, l_{2}, \ldots, l_{k}\right)\left(\operatorname{resp} .\left(l_{1}, l_{2}, \ldots, l_{k}, l\right)\right)$ of length $k$ in $G(\phi)$.

The following well-known lemma provides a necessary and sufficient condition for a 2-SAT formula to be satisfiable. 
Lemma 4.1. (Papadimitriou, 1994) A 2-SAT formula $\phi$ is unsatisfiable iff there is a variable $x$ appearing in $\phi$ such that there is a path from $x$ to $\neg x$ and a path from $\neg x$ to $x$ in $G(\phi)$.

If $\phi$ has a $\delta$-model, then $G(\phi)$ has further restrictions.

Lemma 4.2. If a 2-SAT formula $\phi$ has a $\delta$-model, then there is no path from $l$ to $\neg l$ for any vertex $l$ in $G(\phi)$.

Proof. If there was a path from $l$ to $\neg l$ in $G(\phi)$, then any satisfying assignment has to set $l$ to false. If we now flip the value of the literal $l$ (by flipping the associated variable), we cannot repair to get a model of $\phi$.

Remark. Lemma 4.2 establishes a necessary condition for a satisfiable 2-SAT formula to have a $\delta$-model. Unlike Lemma 4.1, this condition is not sufficient: consider, for example, the 2-SAT formula (which also illustrates many of the constraints that have to be satisfied if a $\delta$-model exists):

$$
\left(v_{1} \rightarrow v_{2}\right) \wedge\left(v_{2} \rightarrow v_{3}\right) \wedge\left(v_{3} \rightarrow v_{4}\right) \wedge\left(v_{4} \rightarrow v_{5}\right) .
$$

Any $\delta$-model of this formula has to set $v_{1}$ to false (otherwise every variable has to be set to true and a break to $v_{5}$ requires more than one repair). Similarly $v_{5}$ has to set to $1, v_{2}$ to 0 and $v_{4}$ to 1 . No choice of $v_{3}$ will allow a single repair to a break to both $v_{1}$ or $v_{5}$. This formula thus does not have a $\delta$-model, yet it satisfies the necessary condition of Lemma 4.2.

We now establish a necessary and sufficient condition for a model of 2-SAT formula $\phi$ to be a $\delta$-model.

Lemma 4.3. Let $\phi$ be a satisfiable 2-SAT formula. Suppose that there is no path from $l$ to $\neg l$ for any vertex $l$ in $G(\phi)$. Let $X$ be a model of $\phi$. Then $X$ is a $\delta$-model if and only if it satisfies the following conditions:

(C1) Let $\mathcal{P}=\left(l_{1}, l_{2}, l_{3}\right)$ be a simple path in $G(\phi)$ of length 2. Then $X\left(l_{1}\right)=0$ and $X\left(l_{3}\right)=1$.

(C2) If $\left(l_{1}, l_{2}\right)$ and $\left(l_{1}, l_{3}\right)$ are edges in $G(\phi)$, then $X\left(l_{1}\right), X\left(l_{2}\right), X\left(l_{3}\right)$ cannot all be 0 .

Proof. $(\Rightarrow)$ Suppose $X$ is a $\delta$-model of $\phi$. Let $P=\left(l_{1}, l_{2}, l_{3}\right)$ be a simple path of length 2 . If $X\left(l_{1}\right)=1$, then $X\left(l_{2}\right)=X\left(l_{3}\right)=1$, otherwise $X$ cannot be a model of $\phi$. A break to $l_{3}$ requires the values of both $l_{1}$ and $l_{2}$ to be flipped so $X$ cannot be a $\delta$-model, a contradiction. So $X\left(l_{1}\right)=0$. Similar arguments show that $X\left(l_{3}\right)=1$. Condition (C2) holds similarly: if $X\left(l_{1}\right), X\left(l_{2}\right), X\left(l_{3}\right)$ were all false, then a break to $l_{1}$ would require two repairs (both $l_{2}$ and $\left.l_{3}\right)$. Hence one of them has to be set to true.

$(\Leftarrow)$ Let $X$ be a model of $\phi$ which satisfies conditions $(\mathrm{C} 1)$ and $(\mathrm{C} 2)$. We show that $X$ is actually a $\delta$-model. Suppose not; say a break to a variable $v$ is not repairable by at most one other bit flip. Assume without loss of generality, that $X(v)=0$ and so after the break, $v$ is set to 1 . There must be at least one clause of the form $v \rightarrow l$ where $l$ is a literal, with $X(l)=0$, otherwise the break does not need a response. If there is more than 1 such clause, say clauses $v \rightarrow l$ and $v \rightarrow l^{\prime}$ with $X(l)=X\left(l^{\prime}\right)=0$, then $X$ violates condition (C2), contradicting the hypothesis. So there is exactly one clause of the form $v \rightarrow l$ with $X(l)=0$ 
and moreover, it must be the case that flipping $l$ does not produce a model of $\phi$ (then one repair would have sufficed). Now since a flip of the variable associated with $l$ repairs the clause $v \rightarrow l$, there must be other clauses that break when $l$ is repaired. Such a clause must be of the form $l \rightarrow l^{\prime}$ for some literal $l^{\prime}$ with $X\left(l^{\prime}\right)=0$. We know that $l^{\prime}$ cannot be $\neg v$ since then we would have a path between $v$ and $\neg v$ in $G(\phi)$, which violates the hypothesis. Our assumption that each clause has distinct literals implies that $l^{\prime} \neq \neg l$. Hence $\left(v, l, l^{\prime}\right)$ is a simple path such that $X(v)=0$ and $X\left(l^{\prime}\right)=0$, contradicting condition (C1). Hence $X$ is a $\delta$-model.

Remark. (i) If $\phi$ has a $\delta$-model, then it is indeed the case that if $(v, u)$ and $(w, u)$ are edges of $G(\phi)$, then $u, v$ and $w$ cannot all be set to true in such a $\delta$-model (since a break to $u$ is not repairable by a single flip). We do not need to include this condition explicitly in Lemma 4.3, because this condition happens if and only if $(\neg u, \neg v)$ and $(\neg u, \neg w)$ satisfy condition (C2) in Lemma 4.3.

(ii) If $\phi$ has a $\delta$-model, then condition (C1) can be extended to specify the values of literals (vertices) on any path of length 3 (the maximum possible length, see Corollary 4.4 below) as follows: if $\left(u_{1}, u_{2}, u_{3}, u_{4}\right)$ is a simple path, then apply condition (C1) twice to get $X\left(u_{1}\right)=X\left(u_{2}\right)=0$ and $X\left(u_{3}\right)=X\left(u_{4}\right)=1$. Thus we do not include this condition explicitly.

Lemma 4.3 has further consequences for $G(\phi)$ :

Corollary 4.4. If a 2-SAT formula $\phi$ has a $\delta$-model, then $G(\phi)$ satisfies the following properties:

(i) The longest simple path in $G(\phi)$ has length at most 3 .

(ii) The longest simple cycle in $G(\phi)$ has length at most 2.

(iii) A vertex $v$ can take part in at most 1 simple cycle.

Proof. Suppose that there is a simple path $\left(l_{1}, l_{2}, l_{3}, l_{4}, l_{5}\right)$ of length 4 in $G(\phi)$. If $X$ is a $\delta$ model of $\phi$, Lemma 4.3 implies that $X\left(l_{3}\right)=1$ when we apply $(\mathrm{C} 2)$ to the segment $\left(l_{1}, l_{2}, l_{3}\right)$ and $X\left(l_{3}\right)=0$ when we apply $(\mathrm{C} 2)$ to the segment $\left(l_{3}, l_{4}, l_{5}\right)$. Hence such a $\delta$-model cannot exist. The other conditions follow from similar arguments.

Pseudo-code for our algorithm is given in Algorithm (1). Observe that Algorithm (1) is a polynomial time reduction from $\delta$-2-SAT to the satisfiability question of a new 2-SAT formula $\phi_{B}$. Proof of correctness follows.

We first need to prove the following easy lemma.

Lemma 4.5. If a 2-SAT formula $\phi$ has a $\delta$-model, then it has a $\delta$-model with each source vertex (respectively, sink vertex) in $G(\phi)$ set to false (resp. true).

Proof. Modify a $\delta$-model $X$ of $\phi$ by setting each sink vertex to 1 (and hence each source vertex to 0 ). Let the new assignment be $X^{\prime}$. Clearly, $X^{\prime}$ is still a model of $\phi$ (setting the antecedent $p$, or the consequent $q$, to 0 , or 1 respectively, satisfies every implication $p \rightarrow q$ ). 


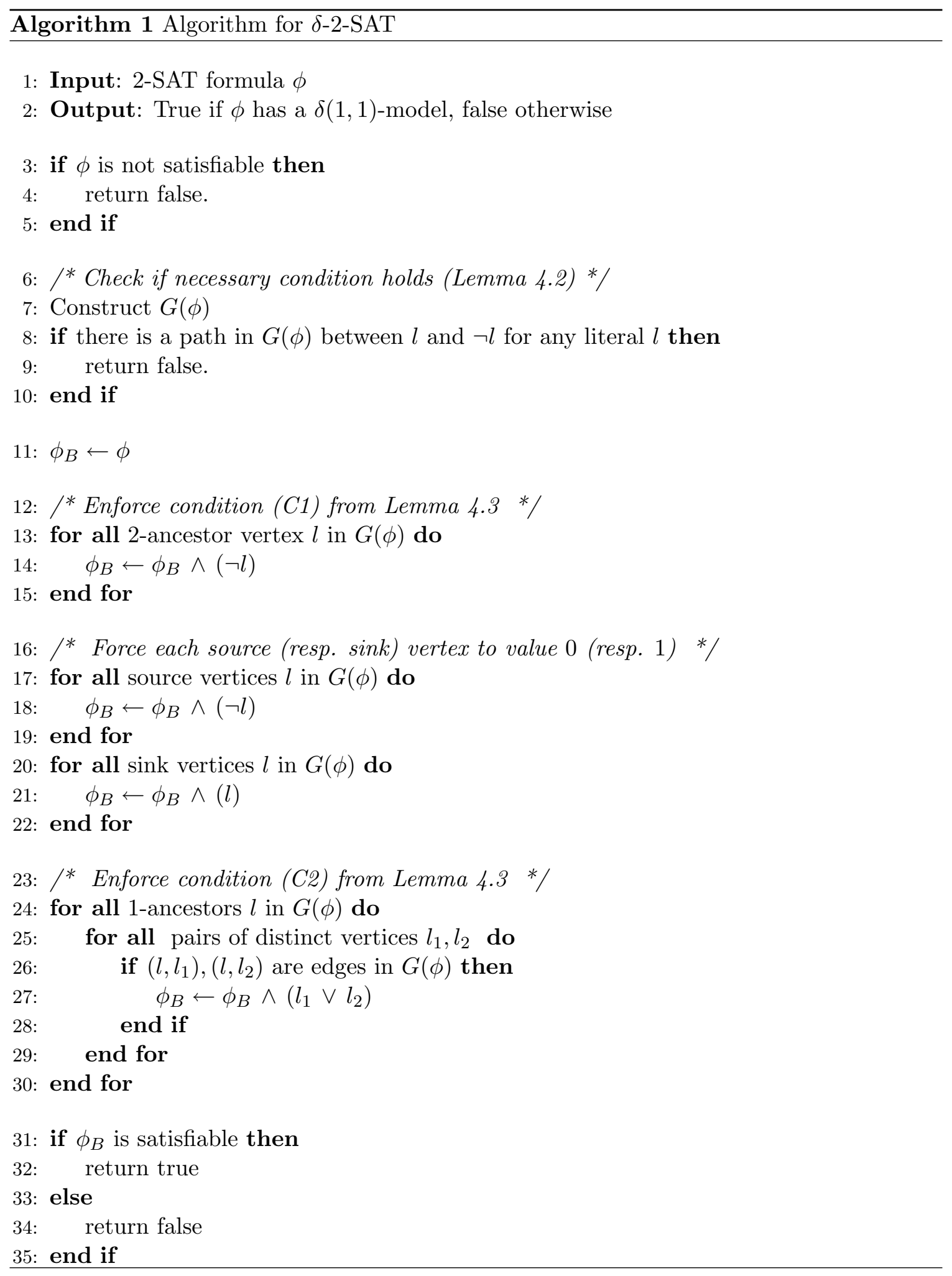


We show that this model satisfies condition $(\mathrm{C} 1)$ and $(\mathrm{C} 2)$ of Lemma 4.3, thus proving that it is a $\delta$-model. If condition (C1) is violated, then there is some simple path $\left(l_{1}, l_{2}, l_{3}\right)$ in $G(\phi)$ where $X^{\prime}\left(l_{1}\right)=1$ or $X^{\prime}\left(l_{3}\right)=0$. If $X^{\prime}\left(l_{1}\right)=1$, then $X\left(l_{1}\right)=1$ (suppose not and let $X\left(l_{1}\right)=0$ : since $\left(l_{1}, l_{2}\right)$ is an edge in $G(\phi), l_{1}$ is not a sink vertex, so its value would not have been changed). Similarly, $X^{\prime}\left(l_{3}\right)=0$ would imply that $X\left(l_{3}\right)=0$. Thus $X$ would violate condition $(\mathrm{C} 1)$ with respect to the simple path $\left(l_{1}, l_{2}, l_{3}\right)$ and could not have been a $\delta$-model (a contradiction). Condition (C2) similarly holds.

Algorithm (1) adds literals to the input 2-SAT formula $\phi$ to enforce variable assignments that must hold if $\phi$ has a $\delta$-model (see Lines 12-15, 24-30 in the body of Algorithm (1)). Since we are guaranteed by Lemma 4.3 that these conditions are a necessary and sufficient condition for the existence of a $\delta$-model, the satisfiability of the resulting Boolean formula would imply that $\phi$ has a $\delta$-model. To simplify the proof of correctness (which is now simply Corollary 4.6 below), we enforce that source and label vertices get default values prescribed by Lemma 4.5 .

Corollary 4.6. The formula $\phi_{B}$ is satisfiable iff $\phi$ has a $\delta$-model.

Proof. Immediate from Lemma 4.3 and Lemma 4.5.

Example 4.1. Let $\phi$ be the 2-SAT formula:

$$
\begin{aligned}
& \left(v_{1} \rightarrow v_{2}\right) \wedge\left(v_{2} \rightarrow v_{3}\right) \\
& \left(v_{1} \rightarrow v_{4}\right) \wedge\left(v_{4} \rightarrow v_{3}\right) \\
& \left(v_{1} \rightarrow v_{5}\right) \wedge\left(v_{5} \rightarrow v_{3}\right)
\end{aligned}
$$

Then Algorithm (1) constructs $\phi_{B}$ where

$$
\begin{gathered}
\phi_{B}=\phi \wedge \\
\left(\neg v_{1}\right) \wedge\left(v_{3}\right) \quad(\text { added by lines 13-16 in Algorithm (1)) } \\
\wedge\left(v_{2} \vee v_{4}\right) \wedge\left(v_{2} \vee v_{5}\right) \wedge\left(v_{4} \vee v_{5}\right) \quad(\text { added by lines 24-31) } \\
\wedge\left(\neg v_{2} \vee \neg v_{4}\right) \wedge\left(\neg v_{2} \vee \neg v_{5}\right) \wedge\left(\neg v_{4} \vee \neg v_{5}\right) \quad \text { (added by lines 24-31) }
\end{gathered}
$$

Note that in the construction of $G(\phi), \neg v_{3}$ is a 2-ancestor. Since two of the variables $v_{2}, v_{4}, v_{5}$ have to be set to the same value, $\phi_{B}$ is unsatisfiable. Hence $\phi$ does not have a $\delta$-model.

Theorem 4.7. In polynomial time, one can determine if a 2-SAT formula has a $\delta$-model and find one if it exists.

Proof. Satisfiability of a 2-SAT formula is in $P$ (Papadimitriou, 1994). Other steps in the procedure consist of looping over simple paths of length 3 , which can be done in time $O\left(n^{3}\right)$ where $n$ is the number of variables.

Remark. It is possible to further characterize the space complexity of $\delta(1,1)$-2-SAT. In fact, $\delta(1,1)-2-\mathrm{SAT}$ is complete for NL (non-deterministic log space). To see that $\delta(1,1)-2$ SAT is in NL, observe that Algorithm (1) can be executed in space logarithmic in the input. Completeness can be established via a log-space reduction from 2-SAT. Since this result is not very relevant in the present context, we leave the details out. 


\subsubsection{An Alternative proof of Theorem 4.7}

An alternative proof of Theorem 4.7 was suggested by one of the reviewers. It is possible to cast any satisfiability problem as a constraint satisfaction problem (CSP) over binary variables. This transformation, particularly when the input instance is a 2-SAT problem, produces a CSP for which local consistency (consistency of subproblems involving fewer variables) ensures the presence of a global solution. In this framework, asserting that a Boolean formula has a $\delta$-model becomes particularly convenient.

Notation: Let $\phi$ be a Boolean formula in CNF. For a subset $S$ of variables, we let $\phi(S)$ denote the subformula of $\phi$ consisting of clauses from $\phi$ which only involve variables in $S$.

Definition 4.1. A formula $\phi$ is said to be $k$-consistent if for every subset $S$ of $k-1$ variables, every model of $\phi(S)$ can be extended to a model of $\phi(S \cup\{v\})$ for every variable $v$ (i.e., a larger subformula of $\phi$ involving one more variable). A formula is strong $k$-consistent if it is $i$-consistent for all $i, 1 \leq i \leq k$.

Remark. The concept of $k$-consistency has other equivalent formulations (Jeavons, Cohen, \& Cooper, 1998; Dechter, 1992). Since our goal in this paper is to study satisfiability exclusively, we rephrase some of the definitions and theorems to apply to our present context.

Theorem 4.8. (Dechter, 1992) Let $\phi$ be a 2-SAT formula. Then the following hold:

(a) If $\phi$ is strong 3 -consistent, then $\phi$ is satisfiable and for any 2 element set $S, \phi(S)$ is satisfiable.

(b) In polynomial time (see e.g., (Jeavons et al., 1998)) one can check whether $\phi$ is strong 3-consistent. If $\phi$ is satisfiable but not strong 3-consistent, then one can add extra clauses (also in 2-CNF) to $\phi$ in polynomial time such that the resulting 2-SAT formula is strong 3 -consistent.

Remark. More generally, given an input Boolean formula $\phi$, one can establish $k$-consistency by adding extra constraints that do not change the set of models. This is done by iterating over all possible $k$-element subsets of variables and solving the subproblem for these variables. Clauses are added which restrict the values of any subset of $k-1$ variables to only those values that can be extended to another variable. If there is a set of $k-1$ variables none of whose assignments can be extended, then we can conclude $\phi$ is unsatisfiable. If not, then these extra clauses are added to $\phi$ to make it $k$-consistent. Enforcing strong $k$-consistency (for fixed $k$ ) can be accomplished in polynomial time (Jeavons et al., 1998; Dechter, 1992).

In the special case when $\phi$ is a 2-SAT formula these extra clauses are also binary and so we end up with a strong 3 -consistent 2-SAT formula (which we denote by $\widehat{\phi}$ ) with exactly the same models (and hence, the same set of $\delta$-models).

Notation: For an ordered pair of variables $(u, v)$, we let $M_{\phi}(u, v)$ denote the set of models of $\phi(\{u, v\})$.

Theorem 4.8 (b) implies that we can assume without loss of generality that the input is a strong 3 -consistent 2-SAT formula $\phi$. Theorem 4.8 also implies that an assignment $X$ 
is a model of $\phi$ iff $(X(u), X(v)) \in M_{\phi}(u, v)$ for all pairs $(u, v)$. Clearly, we can construct all the sets $M_{\phi}(u, v)$ in polynomial time (there are $\Theta\left(n^{2}\right)$ such variable tuples, where $n$ is the number of variables, and each set $M_{\phi}(u, v)$ consists of models of a 2-SAT formula with at most 2 variables). With a slight abuse of notation, we denote $M_{\phi}(-u, v)$ to be the set $\left\{(\neg \alpha, \beta) \mid(\alpha, \beta) \in M_{\phi}(u, v)\right\}$.

Let $u$ be any variable of $\phi$. Let $\phi_{u, 0}=\phi \wedge(\neg u)$ and $\phi_{u, 1}=\phi \wedge(u)$. If either $\phi_{u, 0}$ or $\phi_{u, 1}$ is unsatisfiable, then it is clear that $\phi$ cannot have a $\delta$-model. Assume then that both $\phi_{u, 0}$ and $\phi_{u, 1}$ are satisfiable and let $\widehat{\phi_{u, 0}}$ and $\widehat{\phi_{u, 1}}$ be the corresponding strong 3 -consistent formulas. Let $N_{u}$ be the set of variable pairs $(v, w)$ such that $M_{\widehat{\phi_{u, 0}}}(v, w) \cap M_{\widehat{\phi_{u, 1}}}(v, w)=\emptyset$.

Lemma 4.9. Suppose $N_{u} \neq \emptyset$ for some variable $u$. If $\phi$ has a $\delta$-model, then there is some variable $v$, where $v \neq u$, such that $v$ belongs to every pair in $N_{u}$.

Proof. If we flip the value of $u$ in a $\delta$-model of $\phi$, we can repair by flipping at most one other variable and we are forced to flip one variable from each pair in $N_{u}$. This means that this repair variable is in every pair of $N_{u}$.

Lemma 4.9 implies that we may assume that the pairs in $N_{u}$ have a common member. We can similarly show:

Lemma 4.10. Suppose that $v$ is a variable that appears in every pair in $N_{u}$. Then the following hold:

(i) If there exists a $w$ such that

$$
M_{\widehat{\phi_{u, 0}}}(-v, w) \cap M_{\widehat{\phi_{u, 1}}}(v, w)=\emptyset
$$

then any $\delta$-model $X$ of $\phi$ has to set $X(u)=0$.

(ii) If there exists a $w$ such that

$$
M_{\widehat{\phi_{u, 0}}}(v, w) \cap M_{\widehat{\phi_{u, 1}}}(-v, w)=\emptyset,
$$

then any $\delta$-model $X$ of $\phi$ has to set $X(u)=1$.

Thus either of the two conditions in Lemma 4.10 force the value of the variable $u$ in any $\delta$-model of $\phi$. Together Lemmas 4.9 and 4.10 enable us to set the values of the variables that are forced (cf. Lemma 4.3). If after setting the values of these variables, we derive a contradiction then $\phi$ cannot have a $\delta$-model.

Algorithm (2) provides the detailed description of the algorithm.

Theorem 4.11. Algorithm (2) decides $\delta(1,1)$-2-SAT in polynomial time.

Proof. Enforcing 3-consistency is in polynomial time (Dechter, 1992). The outer loop in Line 3 executes $n$ times where $n$ is the number of variables. Within the body of the loop, calls are made to enforce satisfiability and 3-consistency, along with calls to construct $N_{u}$ for the variable $u$ under consideration. Each step takes polynomial time, hence the claim follows.

Remark. While Algorithm (2) solves the yes/no problem of testing whether an input 2SAT formula has a $\delta$-model, it is a simple matter to modify the algorithm so that it outputs a $\delta$-model if such a model exists. The forced variable assignments along with any satisfying assignment of the remaining 2-SAT formula is a $\delta$-model of the input formula. 


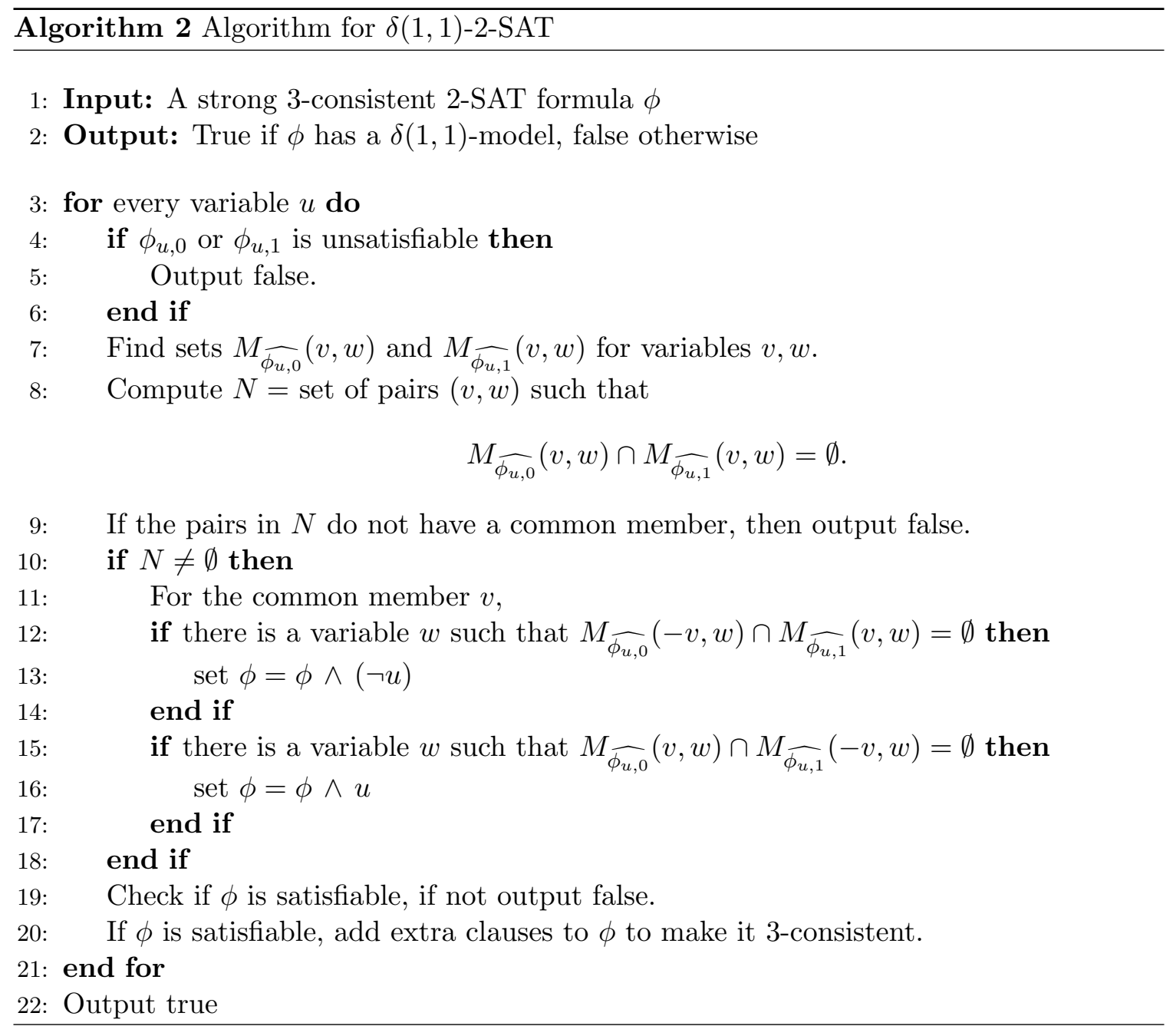




\subsubsection{Complexity of $\delta(1, s)-2$-SAT FOR $s \geq 2$}

Theorem 4.12. The problem $\delta(1, s)$-2-SAT is NP-complete for all $s>1$.

Proof. Clearly this problem is in NP: an NDTM can guess such an assignment and check that it is a model and that for every break, there are at most $s$ other bits that can be flipped to get a model (since $s$ is fixed a priori, this leads to at most $O\left(n^{s}\right)$ possible repair sets, a polynomial number of choices).

We prove NP-completeness via a reduction from $(s+1)$-SAT. Let

$$
T=C_{1} \wedge C_{2} \ldots \wedge C_{m}
$$

be an instance of $(s+1)$-SAT where each clause $C_{i}$ is a disjunction of $s+1$ literals:

$$
v_{i, 1} \vee v_{i, 2} \ldots \vee v_{i, s+1}
$$

We construct an instance $T^{\prime}$ of $\delta(1, s)$-2-SAT as follows: for each clause $C_{i}$ in $T$, we construct an appropriate 2-SAT formula $C_{i}^{\prime}$. Our resulting instance of $\delta(1, s)$-2-SAT is a conjunction of these 2-SAT formulas. Thus,

$$
T^{\prime}=\bigwedge_{1 \leq i \leq m} C_{i}^{\prime}
$$

where $C_{i}^{\prime}$ is a 2-SAT formula defined for each clause $C_{i}$ as follows:

$$
\begin{aligned}
C_{i}^{\prime}= & \bigwedge_{1 \leq j \leq(s+1)}\left(z_{i} \Rightarrow v_{i, j}\right) \\
\bigwedge_{1 \leq j \leq(s+1)}\left(v_{i, j} \Rightarrow \alpha_{i, j, 1}\right) & \bigwedge_{1 \leq j \leq s+1}\left(\alpha_{i, j, k} \Rightarrow \alpha_{i, j, k+1}\right)
\end{aligned}
$$

where we have introduced $1+s(s+1)$ new variables: $z_{i}$ and $\alpha_{i, j, k}$ for $1 \leq j \leq s+1,1 \leq k \leq s$ to define the gadget $C_{i}^{\prime}$. The gadget $C_{i}^{\prime}$ is best understood via Figure (1).

Let $T$ have a model $X$. Extend that to a model of $T^{\prime}$ by setting $z_{i}=0$ for all $1 \leq i \leq m$ and $\alpha_{i, j, k}=1$ for all $1 \leq i \leq m, 1 \leq j \leq s+1,1 \leq k \leq s$. We claim that this is a $\delta(1, s)$-model of $T^{\prime}$. Suppose we flip the variable corresponding to literal $l$. Now we do a case analysis of how many repairs are needed:

- $\left[l=z_{i}\right]$ Since $v_{i, 1} \vee v_{i, 2} \ldots \vee v_{i, s+1}$ is set true by the model $X$, we need to flip at most $s$ false literals in $\left\{v_{i, 1}, \ldots, v_{i, s+1}\right\}$. Observe that no more repairs are necessary.

- $\left[l=\alpha_{i, j, k}\right]$ Need to flip $\alpha_{i, k, k^{\prime}}$ where $1 \leq k^{\prime}<k$ and we might need to flip the variable corresponding to $v_{i, j}$ if $v_{i, j}$ was set to true by $X$. This repair does not affect the truth value of other clauses of $T^{\prime}$. Hence we flip at most $s$ variables.

- $[l=$ variable occurring in $T]$ This will flip the value of all literals involving $l$. Because we set every $a_{i, j, k}=1$ and $z_{i}=0$, no repairs are needed in $T^{\prime}$, as each implication (clause) of $T^{\prime}$ still remains true. 


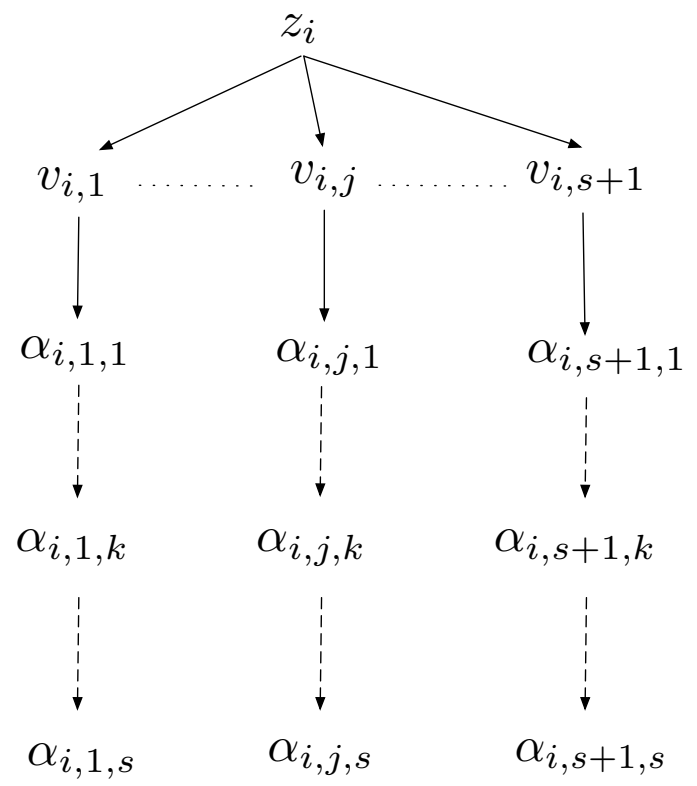

Figure 1: Gadget for 2-SAT

Now suppose $T^{\prime}$ has a $\delta(1, s)$-model. We show that $T$ has a model. Note that in such a model $z_{i}=0$ for all $i$ (otherwise if $z_{i}=1$, then $v_{i, j}=\alpha_{i, j, k}=1$ and we will need more than $s$ repairs when we flip the value of $\left.\alpha_{i, 1, s}\right)$. Now all literals $\left\{v_{i, 1}, v_{i, 2}, \ldots, v_{i, s+1}\right\}$ cannot be set to 0 , since a break to $z_{i}$ would again necessitate $s+1$ repairs. Hence at least one of the literals in $\left\{v_{i, 1}, v_{i, 2}, \ldots, v_{i, s+1}\right\}$ is set to 1 . In other words, the clause $C_{i}$ in $T$ is satisfied. Since $z_{i}=0$ for all $i, T$ must have a model.

\subsubsection{Complexity of $\delta^{*}-2-\mathrm{SAT}$}

In this section, we show that $\delta^{*}-2-\mathrm{SAT}$ is in polynomial time.

Let $\phi$ be the input 2-SAT formula over $n$ variables. We construct the graph $G(\phi)$ as described before in Section 4.1.1. Since a $\delta^{*}$-model is by definition also a $\delta$-model, we must have the same path restrictions set forth by Lemma 4.3 and Lemma 4.2 . If $\phi$ has a $\delta^{*}$-model, then $G(\phi)$ has further restrictions.

Lemma 4.13. Let $\phi$ be a 2-SAT formula with a $\delta^{*}$-model. Then every non-trivial simple path in $G(\phi)$ has length 1.

Proof. Suppose that $\left(l_{1}, l_{2}, l_{3}\right)$ is a simple path in $G(\phi)$ of length 2 . Let $X$ be a $\delta^{*}$-model of $\phi$. Because of Lemma 4.3, we know that $X\left(l_{1}\right)=0, X\left(l_{3}\right)=1$ and this has to be the case for all $\delta^{*}$-models. This means that a break to $X\left(l_{1}\right)$ cannot be repaired to get another $\delta^{*}$-model. Hence, $X$ cannot be a $\delta^{*}$-model, a contradiction. 
Remark. Note $G(\phi)$ may have cycles $\left(l_{1}, l_{2}, l_{1}\right)$, however in that situation, Lemma 4.13 implies that $\left\{l_{1}, l_{2}\right\}$ must form one connected component. Any $\delta^{*}$-model if it exists assigns the same value to $l_{1}$ and $l_{2}$ such that the respective variables form a break-repair pair and are independent of the remaining variables. We can thus remove the cycles from consideration. So without loss of generality, we assume that $G(\phi)$ has no cycles.

Let $R$ be the vertices in $G(\phi)$ with in-degree 0 and $B$ be the vertices with out-degree 0 . Since a vertex cannot have positive in-degree and positive out-degree, this creates a bipartition $R \cup B$ of the vertices of $G(\phi)$, where $R, B$ are disjoint vertex sets and all edges in $G(\phi)$ are of the form $\left(l, l^{\prime}\right)$ with $l \in R$ and $l^{\prime} \in B$.

Note that if $\left(l, l^{\prime}\right)$ is an edge in $G(\phi)$, then the out-degree of $\neg l$ is 0 : otherwise, there would be a path of length 2 or a cycle, both of which we have excluded. Hence $l \in R$ iff $\neg l \in B$. We also observe that there are no isolated vertices in $G(\phi)$ since every clause is a disjunction of distinct literals. This gives a complete graph theoretic characterization of the structure of $G(\phi)$ when $\phi$ has a $\delta^{*}$-model.

Now let $Y_{0}$ be an assignment that sets every literal in $R$ false (0) and (hence sets) every literal in $B$ true (since we have assumed that every variable appears in both positive and negative literals).

Lemma 4.14. The assignment $Y_{0}$ is a $\delta^{*}$-model.

Proof. We exhibit a stable set $\mathcal{C}$ of models of $\phi$ that contains $Y_{0}$. Let $Y \downarrow_{B}$ (respectively, $\left.Y\right|_{R}$ ) denote the restriction of an assignment $Y$ onto the literals in $B$ (respectively, $R$ ).

Let

$$
\mathcal{C}=\left\{Y \mid Y \iota_{B} \text { contains at most one literal set false }\right\} .
$$

Note that if $Y \downarrow_{B}$ contains at most one false literal, then $Y l_{R}$ contains at most one true literal. Clearly $Y_{0} \in \mathcal{C}$.

We now show that $\mathcal{C}$ is a stable set. Let $Y \in \mathcal{C}$, where $Y \neq Y_{0}$. Suppose that $Y$ sets the literal $l \in R$ to true and $\neg l$ to false in $B$. If the value of the literal $l$ is flipped, then we get $Y_{0}$ (a model in $\mathcal{C}$ ) and so no repairs are needed. If a different variable is flipped, then this creates a new literal $l^{\prime}$ in $R$ set to true (and $\neg l^{\prime}$ false in $B$ ) in the new assignment. Then we repair by flipping the value of $l$ from true to false, thereby allowing only one positive literal in $R$. Thus any break to $Y$ is repairable by another model in $\mathcal{C}$. A break to $Y_{0} \in \mathcal{C}$ does not need any repairs. Hence $\mathcal{C}$ is a stable set and $Y_{0}$ is a $\delta^{*}$-model.

Theorem 4.15. $\delta^{*}-2-S A T \in P$.

Proof. The graph $G(\phi)$ can be constructed in polynomial time (in time linear in the size of $\phi)$. All conditions needed for the existence of a $\delta$-model can be checked in polynomial time: using depth-first search, one can check if the longest simple path of $G(\phi)$ has length 1 and check whether the subgraph of $G$ without any 2-cycles is bipartite.

\subsection{Finding $\delta$-models for Horn-SAT and dual Horn-SAT}

Recall that an instance of Horn-SAT is a Boolean formula in CNF where each clause contains at most 1 positive literal. As in 2-SAT, there is a polynomial time algorithm to find a model 
of a Horn formula (see, e.g., Papadimitriou (1994)). However, unlike the situation in 2$\mathrm{SAT}$, finding $\delta(1, s)$-models for Horn formulas is NP complete for all $s \geq 1$. The proof of this fact can be easily modified to show that the same problem is NP-complete for dual Horn-SAT.

We first prove a technical lemma which will be used in the NP-completeness proof. Define the Boolean formula $\phi=\phi\left(x, y, \beta_{1}, \ldots, \beta_{2 s}\right)$ over variables $x, y, \beta_{1}, \ldots, \beta_{2 s}$ as follows:

$$
\begin{aligned}
\phi\left(x, y, \beta_{1}, \ldots, \beta_{2 s}\right)= & \bigwedge_{i=1}^{s-1}\left(\beta_{i} \Rightarrow \beta_{i+1}\right) \\
& \wedge\left(\beta_{s} \Rightarrow x\right) \wedge\left(\beta_{s} \Rightarrow y\right) \\
& \wedge\left(x \Rightarrow \beta_{s+1}\right) \wedge\left(y \Rightarrow \beta_{s+1}\right) \wedge \\
& \bigwedge_{i=s+1}^{2 s-1}\left(\beta_{i} \Rightarrow \beta_{i+1}\right)
\end{aligned}
$$

The formula $\phi$ is best visualized as in Figure (2). Observe that each variable $x$ and $y$ appears both as the head and tail of a chain of implications of length $s$.

Figure 2: Gadget $\phi$

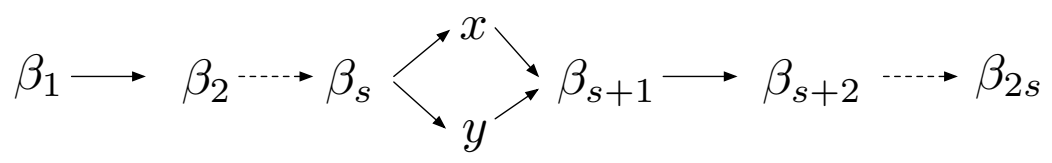

The crucial property of this gadget that we use is as follows:

Lemma 4.16. Let $X$ be a model of $\phi$. Then $X$ is a $\delta(1, s)$-model iff it satisfies $x \Leftrightarrow \neg y$.

Proof. $(\Leftarrow)$ Let $X$ be a model of $\phi$. If $x \Leftrightarrow \neg y$ holds for $X$ (i.e., $x$ and $y$ get opposite truth values in $X$ ), then $X$ has to set all $\beta_{i}$ with $i \leq s$ to 0 (because either $x$ and $y$ is set to 0 ) and all $\beta_{j}$ with $j \geq s+1$ to true (because either $x$ or $y$ is set to 1 ). Then a break to $\beta_{i}$ with $i \leq s$ requires repairs to $\beta_{j}$ where $i<j \leq s$ and exactly one of $x$ and $y$ (the variable set to 0$)$. Similarly a break to $\beta_{i}$ with $i \geq s+1$ requires repairs to $\beta_{j}, s+1 \leq j \leq i-1$ and exactly one of $x$ and $y$ (the variable set to 1 ). A break to $x$ or $y$ does not need any repairs. Since we never need more than $s$ repairs for every break, $X$ is a $\delta(1, s)$-model.

$(\Rightarrow)$ Any $\delta(1, s)$-model $X$ of $\phi$ has to set each $\beta_{j}$ to 0 where $1 \leq j \leq s$ and each $\beta_{j}$ to 1 where $s+1 \leq j \leq 2 s$ (otherwise more than $s$ repairs are needed for breaks to these variables). If both $X(x)=X(y)=0$, then a break to $\beta_{1}$ (from a 0 to 1 ) would require repairs to $\beta_{2}, \beta_{3}, \ldots, \beta_{s}$ as well as to both $x$ and $y$, for a total of $s+1$ repairs. Hence both $x$ and $y$ cannot be false. Similarly, both $x$ and $y$ cannot be true because then a break to $\beta_{2 s}$ would require $s+1$ repairs. Hence $X$ satisfies $x \Leftrightarrow \neg y$.

Theorem 4.17. $\delta(1, s)$-Horn-SAT is NP-complete for $s \geq 1$. 
Proof. We prove this via a reduction from 3-SAT. Let $T$ be an instance of 3-SAT, where $T=\bigwedge_{i=1}^{m} C_{i}$ is defined over $n$ variables $x_{1}, x_{2}, \ldots, x_{n}$ and clause $C_{i}$ is a disjunction of 3 distinct literals. Clearly we can assume that every variable appears in both positive and negative literals in $T$ (if not, we may set the pure literal to be true or false appropriately and consider the resulting formula as $T$ ).

We first apply an intermediate transformation to $T$. We replace any positive literal (say $x_{j}$ ) in $C_{i}$ by a negative literal, $\neg x_{j}^{\prime}$, where $x_{j}^{\prime}$ is a new variable not occurring in $T$. The new clause, which now has no positive literal, is denoted by $C_{i}^{\prime}$. Remembering our global assumption that every variable in input Boolean formulas appear in both positive and negative literals, we see that this transformation will introduce variables $x_{j}^{\prime}$ for every variable $x_{j}$ in $T$. To maintain logical equivalence, we also need to enforce that $\neg x_{j}^{\prime} \Leftrightarrow x_{j}$ in the new formula: so we add the following clauses: $\left(\neg x_{j}^{\prime} \vee \neg x_{j}\right)$ and $\left(x_{j}^{\prime} \vee x_{j}\right)$. Note that these two clauses imply that in any model of this new Boolean formula, $x_{j}$ and $x_{j}^{\prime}$ cannot have the same truth value.

Thus we obtain

$$
T^{\prime}=\bigwedge_{1 \leq i \leq m} C_{i}^{\prime} \bigwedge_{1 \leq i \leq n}\left\{\left(\neg x_{i}^{\prime} \vee \neg x_{i}\right) \wedge\left(x_{i}^{\prime} \vee x_{i}\right)\right\}
$$

Note that $T^{\prime}$ is almost Horn (since every clause $C_{i}^{\prime}$ is Horn), the only non-Horn clauses are the clauses of the form $\left(x_{i} \vee x_{i}^{\prime}\right)$. We have introduced $n$ new variables and $2 n$ new clauses, so that $T^{\prime}$ has $m+2 n$ clauses and is defined over $2 n$ variables. Clearly $T^{\prime}$ is satisfiable iff $T$ is satisfiable.

We now construct an instance $T^{\prime \prime}$ of $\delta(1, s)$-Horn-SAT from $T^{\prime}$ such that $T^{\prime \prime}$ has a $\delta(1, s)$ model iff $T^{\prime}$ is satisfiable. We first introduce $s+1$ new variables $A_{1}, A_{2}, \ldots, A_{s+1}$. For each clause $C_{i}^{\prime}=\neg v_{i, 1} \vee \neg v_{i, 2} \vee \neg v_{i, 3}$, we construct a formula $\Gamma_{i, 1}$ consisting of a single clause (note that at this step, each $v_{i, j}$ is a variable of the form $x_{k}$ or of the form $x_{k}^{\prime}$ for some $k, 1 \leq k \leq n)$ :

$$
\Gamma_{i, 1}=\left(\neg z_{i} \vee \neg w_{i, 1} \vee \neg w_{i, 2} \vee \neg w_{i, 3}\right)
$$

where $z_{i}, w_{i, 1}, w_{i, 2}, w_{i, 3}$ are new variables introduced for each clause $C_{i}^{\prime}$. This step introduces 4 new variables per clause $C_{i}^{\prime}$ for a total of $4 m$ new variables. Our next step creates formulas that places restrictions on these new variables and ties them in with the variables $v_{i, j}$ in the original clause. We introduce new variables $\alpha_{i, j, k}$ for each clause $C_{i}^{\prime}$, where $1 \leq j \leq 3,1 \leq k \leq s-1$, these variables forming the intermediate variables in a chain of implications of length $s$ from $v_{i, j}$ to $w_{i, j}$ as below:

$$
\begin{aligned}
\Gamma_{i, 2} & =\left(v_{i, 1} \Rightarrow \alpha_{i, 1,1}\right) \wedge\left(\alpha_{i, 1,1} \Rightarrow \alpha_{i, 1,2}\right) \cdots \wedge\left(\alpha_{i, 1, s-1} \Rightarrow w_{i, 1}\right) \\
& \wedge\left(v_{i, 2} \Rightarrow \alpha_{i, 2,1}\right) \wedge\left(\alpha_{i, 2,1} \Rightarrow \alpha_{i, 2,2}\right) \cdots \wedge\left(\alpha_{i, 2, s-1} \Rightarrow w_{i, 2}\right) \\
& \wedge\left(v_{i, 3} \Rightarrow \alpha_{i, 3,1}\right) \wedge\left(\alpha_{i, 3,1} \Rightarrow \alpha_{i, 3,2}\right) \cdots \wedge\left(\alpha_{i, 3, s-1} \Rightarrow w_{i, 3}\right)
\end{aligned}
$$

The reader may wish to compare the the gadget $\Gamma_{i, 2}$ with a similar gadget $C_{i}^{\prime}$ in Equation (4.1) and shown in Figure (1) that was used in the proof of Theorem 4.12. 
We also make $z_{i}$, one of the new variables introduced in $\Gamma_{i, 1}$, appear as the head of a chain of implications of length $s+1$ as shown below in formula $\Gamma_{i, 3}$ :

$$
\Gamma_{i, 3}=\left(z_{i} \Rightarrow A_{1}\right) \wedge\left(A_{1} \Rightarrow A_{2}\right) \ldots \wedge\left(A_{s} \Rightarrow A_{s+1}\right)
$$

We now define the formula $C_{i}^{\prime \prime}$ constructed for each clause $C_{i}^{\prime}, 1 \leq i \leq m$, of $T^{\prime}$ :

$$
C_{i}^{\prime \prime}=\Gamma_{i, 1} \wedge \Gamma_{i, 2} \wedge \Gamma_{i, 3}
$$

Note that each $C_{i}^{\prime \prime}$ is Horn and has introduced new variables $\alpha_{i, j, k}, w_{i, j}, z_{i}$ for a total of $3(s-1)+3+1=3 s+1$ new variables. The other new variables $A_{i}$ are global, i.e, reused in the formulas for $C_{i}^{\prime \prime}$ for various $i$.

For the clauses of the form $\left(\neg x_{i}^{\prime} \vee \neg x_{i}\right) \wedge\left(x_{i}^{\prime} \vee x_{i}\right)$ from $T^{\prime}$, where $1 \leq i \leq n$, we introduce new variables $\beta_{i, j}$ for each $i$ where $1 \leq j \leq 2 s$ and construct the gadget $\phi_{i}=$ $\phi\left(x_{i}, x_{i}^{\prime}, \beta_{i, 1}, \beta_{i, 2}, \ldots, \beta_{i, 2 s}\right)$ defined in Equation (4.2).

Our instance of $\delta(1, s)$-Horn-SAT is then:

$$
T^{\prime \prime}=\bigwedge_{1 \leq i \leq m} C_{i}^{\prime \prime} \wedge \bigwedge_{1 \leq i \leq n} \phi_{i}
$$

We first show that if $T^{\prime}$ is satisfiable, then $T^{\prime \prime}$ has a $\delta$-model. Suppose $T^{\prime}$ had a model $X^{\prime}$. Extend that to an assignment $X^{\prime \prime}$ of the variables of $T^{\prime \prime}$ by setting the values of the newly introduced variables as follows:

$$
\begin{gathered}
A_{i}=1 \text { for } 1 \leq i \leq s+1, \\
\qquad z_{i}=0 \text { for } 1 \leq i \leq m, \\
w_{i, j}=1 \text { for all } i \text { and } j \text {, where } 1 \leq i \leq m \text { and } 1 \leq j \leq 3, \\
\alpha_{i, j, k}=1 \text { for all } i, j \text { where } 1 \leq i \leq m, 1 \leq j \leq 3,1 \leq k \leq s-1, \\
\beta_{i, j}=0 \text { for all } j, 1 \leq j \leq s \text { and all } i, 1 \leq i \leq n, \\
\beta_{i, j}=1 \text { for all } j, s+1 \leq j \leq 2 s \text { and all } i, 1 \leq i \leq n .
\end{gathered}
$$

Since $X^{\prime \prime}$ satisfies each clause in $T^{\prime \prime}$, it is a model of $T^{\prime \prime}$. We now show that $X^{\prime \prime}$ is actually a $\delta(1, s)$-model. Suppose that some variable $v$ of $T^{\prime \prime}$ is flipped. We do a case by case analysis of the possible repairs to this break.

$\left[v=x_{i}\right.$ or $\left.x_{i}^{\prime}\right]$ No repairs are needed since each implication remains satisfied in $T^{\prime}$.

[ $v=A_{i}$ for some $\left.i, 1 \leq i \leq s+1\right]$ The repairs needed are $A_{1}, A_{2}, \ldots, A_{i-1}$ (since $z_{i}=0$ for all $i$, it does not need to be flipped) for $i-1(\leq s)$ repairs.

$\left[v=\beta_{i, j}\right.$ for some $\left.1 \leq i \leq n, 1 \leq j \leq s\right]$ The repairs are all $\beta_{i, k}$ where $j+1 \leq k \leq s$. Since $X^{\prime}$ is a model of $T^{\prime}$, exactly one of $x_{i}$ and $x_{i}^{\prime}$ is set to false and we need to flip just that variable. This leads to at most $s-j+1 \leq s$ repairs.

$\left[v=\beta_{i, j}\right.$ for some $\left.1 \leq i \leq n, s+1 \leq j \leq 2 s\right]$ The repairs needed are $\beta_{i, k}$ for all $s+1 \leq$ $k<j$ and one of $x_{i}$ or $x_{i}^{\prime}$ (since $X^{\prime}$ is a model of $T^{\prime}$ only one of $x_{i}, x_{i}^{\prime}$ is set to true in $X^{\prime}$ ) for at most $j-s \leq s$ repairs. 
$\left[v=w_{i, j}\right.$ for some $\left.1 \leq i \leq m, 1 \leq j \leq 3\right]$ The repairs needed are $\alpha_{i, j, k}$ for all $1 \leq k \leq s-1$ and $v_{i, j}$ (if $X^{\prime}\left(v_{i, j}\right)=1$ ), for at most $s$ repairs.

[ $v=\alpha_{i, j, k}$ for some $\left.1 \leq i \leq m, 1 \leq j \leq 3,1 \leq k \leq s-1\right]$ The repairs needed are $\alpha_{i, j, k^{\prime}}$ for $1 \leq k^{\prime} \leq k-1$ and $v_{i, j}$ (if $X^{\prime}\left(v_{i, j}\right)=1$ ) for at most $k \leq s-1$ repairs.

$\left[v=z_{i}\right]$ It is this break alone whose repair crucially depends on the satisfiability of $T^{\prime}$. Note that this break changes $z_{i}$ from a 0 to a 1 and makes the clause $\left(\neg z_{i} \vee \neg w_{i, 1} \vee\right.$ $\left.\neg w_{i, 2} \vee \neg w_{i, 3}\right)$ false since each $w_{i, j}$ is true in $X^{\prime \prime}$. So repairs will have to include one or more of the $w_{i, j}$ 's, which consequently might trigger flips to $\alpha_{i, j, k}$ and $v_{i, j}$. The choice of which $w_{i, j}$ to involve in the repair process is indicated by the $v_{i, j}$ set to 0 by $X^{\prime}$. Since $X^{\prime}$ is a model, note also that at least one $v_{i, j}$ is set to 0 . Without loss of generality, assume that $X^{\prime}\left(v_{i, 1}\right)=0$ then repair a break to $z_{i}$ by flipping $w_{i, 1}, \alpha_{i, 1, j}$ for all $1 \leq j \leq s-1$ for exactly $s$ repairs.

Now suppose $T^{\prime \prime}$ has a $\delta$-model $X^{\prime \prime}$. We show that $T^{\prime}$ is satisfiable. Specifically, we claim that the restriction of $X^{\prime \prime}$ to the variables of $T^{\prime}$ is a model of $T^{\prime}$. From Lemma 4.16, we know that $\beta_{i, j}=0$ for all $1 \leq i \leq n, 1 \leq j \leq s$ and $\beta_{i, j}=1$ for all $1 \leq i \leq n, s+1 \leq j \leq 2 s$ and also $\neg x_{i}^{\prime} \Leftrightarrow x_{i}$ in $T^{\prime}$ is satisfied for each $i, 1 \leq i \leq n$. Note that in $T^{\prime \prime}, w_{i, j}$ is at the end of a chain of implications:

$$
\beta_{k, 1} \rightarrow \beta_{k, 2} \rightarrow \cdots \rightarrow \beta_{k, s} \rightarrow v_{i, j} \rightarrow \alpha_{i, j, 1} \rightarrow \cdots \rightarrow \alpha_{i, j, s-1} \rightarrow w_{i, j}
$$

where $v_{i, j}$ is either $x_{k}$ or $x_{k}^{\prime}$ for some $k, 1 \leq k \leq n$. Note that the variables in the above chain are from different gadgets - from both $\phi_{k}$ and from $\Gamma_{i, 2}$. This implies that $X^{\prime \prime}\left(w_{i, j}\right)=1$ since otherwise $X^{\prime \prime}$ would have to set all variables in this chain to 0 and then this would violate Lemma 4.16. Since $X^{\prime \prime}$ is a model of $T^{\prime \prime}$, it must be that $X^{\prime \prime}\left(z_{i}\right)=1$ for all $i$, otherwise $\neg z_{i} \vee \neg w_{i, 1} \vee \neg w_{i, 2} \vee \neg w_{i, 3}$ will be false. When $z_{i}$ is flipped, we are guaranteed a repair of at most $s$ flips that will make the clause $\neg z_{i} \vee \neg w_{i, 1} \vee \neg w_{i, 2} \vee \neg w_{i, 3}$ true. This will involve flipping at least one of $w_{i, j}$, for $j=1,2,3$. If $v_{i, 1}, v_{i, 2}$ and $v_{i, 3}$ were all set to true by $X^{\prime \prime}$ (which would in turn have implied that $X^{\prime \prime}\left(\alpha_{i, j, k}\right)=1$ for all $1 \leq j \leq 3,1 \leq k \leq s-1$ ) then any such flip would require $s$ additional repairs, for a total of $s+1$ repairs to a break to $z_{i}$. So it must be that $v_{i, j}$ is false for some $j, 1 \leq j \leq 3$. In other words, $C_{i}^{\prime}=\neg v_{i, 1} \vee \neg v_{i, 2} \vee \neg v_{i, 3}$ is satisfied by $X^{\prime \prime}$. Hence the restriction of $X^{\prime \prime}$ to $T^{\prime}$ satisfies all clauses of $T^{\prime}$. Thus $T^{\prime}$ is satisfiable.

So $T^{\prime}$ is satisfiable iff $T^{\prime \prime}$ has a $\delta(1, s)$-model. Since $T$ is satisfiable iff $T^{\prime}$ is satisfiable and $T$ is a SAT instance, this accomplishes the reduction from SAT. This reduction is clearly a polynomial time reduction. Since $\delta(1, s)$-Horn-SAT is clearly in NP for fixed $r$ and $s$, this proves that it is NP-complete.

Recall that an dual-Horn formula is a Boolean formula in CNF where each clause has at most one negative literal. Not surprisingly, dual-Horn-SAT formulas behave similarly to Horn-SAT when it comes to finding $\delta$-models.

Theorem 4.18. $\delta(1, s)$-dual-Horn-SAT is NP-complete.

The proof of this theorem is very similar to that of Theorem 4.17: we replace Equation (4.3) by $\Gamma_{i, 1}=\left(z_{i} \vee w_{i, 1} \vee w_{i, 2} \vee w_{i, 3}\right)$ and change the direction of implications in $\Gamma_{i, 3}$ and Equation (4.4). 


\subsection{Finding $\delta$-models for 0-valid, 1-valid SAT formulas}

Recall that a 0-valid (resp. 1-valid) Boolean formula is one which is satisfied by a model with every variable set to 0 (resp. 1). We now consider the complexity of finding faulttolerant models of an input 0-valid (or 1-valid) formula and refer to the corresponding decision questions as $\delta(r, s)$-0-valid-SAT, $\delta(r, s)$-1-valid-SAT, $\delta^{*}$-0-valid-SAT etc.

The knowledge that an input Boolean formula is satisfied by some particular assignment does not provide information about the presence of fault-tolerant models. Hence we would expect (correctly) that finding such models to be NP-hard. We first prove:

Theorem 4.19. The decision problem $\delta(r, s)$-0-valid-SAT is NP-complete.

Proof. For the proof, we refer to the proof of Theorem 3.1 which, with slight modification, works for this problem as well. We reduce from SAT. Let $T$ be a SAT instance, we construct an instance of $\delta(r, s)$-0-valid-SAT, $T^{\prime}=T \vee \neg y$ where $y$ is a new variable not appearing in $T$. Observe that $T^{\prime}$ is 0 -valid (its the value of $y$ that matters). The proof that $T^{\prime}$ has a $\delta$-model iff $T$ is satisfiable is identical to the proof of Theorem 3.1: if $T$ is satisfiable and has a model $X$, extend that to a model $X^{\prime}$ of $T^{\prime}$ by setting the value of $y$ to 1 . Then any break consisting of $r$ variables in $X^{\prime}$ does not require a repair if the $r$ variables involve $y$. If they do not involve $y$, then flipping the value of $y$ from a 1 to a 0 makes $T^{\prime}$ true, hence one repair suffices. Hence $X^{\prime}$ is a $\delta(r, s)$-model. If $T^{\prime}$ has a $\delta(r, s)$-model, it must have a model with $y$ set to 1 . The restriction of that model to the variables of $T$ makes $T$ true, hence $T$ is satisfiable.

Similarly, it is easy to verify that the proofs of Theorem 3.1, Theorem 3.2 work when the input formula is a 0 -valid or 1-valid formula. Hence we have the following:

Theorem 4.20. The decision problem $\delta(r, s)$-1-valid-SAT is NP-complete. The problem $\delta^{*}(1,1)$-0-valid-SAT and $\delta^{*}(1,1)$-1-valid-SAT are in NEXP and are NP-hard.

\subsection{Finding $\delta$-models for Affine-SAT}

Another class of Boolean formulas that have polynomial time satisfiability checkers is AffineSAT: these are formulas which are a conjunction of clauses, where each clause is an exclusiveor (denoted by $\oplus$ ) of distinct literals $(a \oplus b=1$ iff exactly one of the Boolean variables $a, b$ is set to 1$)$.

Example 4.2. An example of an Affine-SAT formula is

$$
\left(x_{1} \oplus x_{2} \oplus x_{3} \oplus x_{4}=1\right) \wedge\left(x_{3} \oplus x_{4}=0\right)
$$

This formula has a $\delta$-model $X=(1,0,0,0)$. In fact, $X$ is easily seen to be a $\delta^{*}$-model (which is true of all $\delta$-models of Affine-SAT formulas, as we shall shortly see).

One can find a satisfying assignment for a formula in affine form by a variant of Gaussian elimination. We now prove that finding $\delta$-models for affine formulas is also in polynomial time.

Lemma 4.21. An Affine-SAT formula $\phi$ has a $\delta$-model iff $\phi$ is satisfiable and for every variable $v \in V$ appearing in $\phi$ there exists a variable $w=w(v)$ such that $v$ and $w$ appear in exactly the same clauses. 
Proof. Let $X$ be a $\delta$-model of $\phi$. If a variable $v$ is flipped, then the clauses that $v$ appears in become false, to repair them we need to flip some other variable that appears in exactly those clauses (and no others). Thus such a variable pairing must exist. The reverse direction is easily proved: if such a variable pairing exists, then the variables form a break-repair pair.

Since the conditions of Lemma 4.21 are easy to check in polynomial time, we have the following theorem:

Theorem 4.22. $\delta(1,1)$-Affine-SAT $\in P$.

We can, in fact, slightly strengthen our theorem. We first state an analogue of Lemma 4.21, where the variable pairings can be easily generalized.

Definition: The parity of an integer $n$ is $n \bmod 2$.

Lemma 4.23. An Affine-SAT formula $\phi$ has a $\delta(r, s)$-model iff $\phi$ is satisfiable and for every set $R$ of at most $r$ variables, there exists a set $S, S \cap R=\emptyset$ of at most $s$ variables, such that for all clauses $C$ of $\phi$, the parity of the number of variables of $R$ appearing in $C$ is the same as the parity of the number of variables of $S$ appearing in $C$.

We now prove:

Theorem 4.24. $\delta(r, s)$-Affine-SAT is in $P$.

Proof. Since $r$ and $s$ are fixed constants, the conditions in Lemma 4.23 can be checked in polynomial time: for each choice of the set $R$ such that $R \leq r$, (there are $O\left(n^{r}\right)$ such sets), cycle through each possible set $S$ where $|S| \leq s, S \cap R=\emptyset$ (there are $O\left(n^{s}\right)$ such sets), check to see if the conditions of Lemma 4.23 are satisfied (in particular, test whether the parity of the variables of $R$ appearing in any clause $=$ parity of the variables of $S$ appearing in the clause, which also can be accomplished in polynomial time).

Hence $\delta(r, s)$-Affine-SAT is in polynomial time.

Theorem 4.22 implies that any $\delta$-model of $\phi$ is actually a $\delta^{*}$-model, since if the pairings $(u, w(v))$ exist, any model of $\phi$ will become a $\delta$-model (with $\{v, w(v)\}$ forming break-repair pairs).

Hence an Affine-SAT formula has a $\delta$-model iff it has a $\delta^{*}$-model, hence finding a $\delta^{*}$ model for Affine-SAT formulas is also in polynomial time.

We thus have the following theorem:

Theorem 4.25. $\delta^{*}$-Affine-SAT $\in P$.

\section{Future Work}

The complexity of $\delta(r, s)$-SAT where $r$ and $s$ are part of the input as opposed to being fixed constants is not known. This problem is in the complexity class $\Sigma_{3}^{p}$, but is it complete for that class? The status of this problem for restricted Boolean formulas like 2-SAT, HornSAT etc., when $r$ and $s$ are specified in the input is similarly open. At present, we do not also know if $\delta^{*}(r, s)$-SAT can be decided in polynomial space when $r, s$ are fixed constants.

Finally, a practical modification of the concept of $\delta$-models would involve weakening the condition to allow for only a high percentage of breaks to be repairable. 


\section{Acknowledgements}

The author is grateful to Eugene M. Luks for his encouragement and advice. We also thank the anonymous referees for their detailed comments and suggestions.

\section{References}

Bailleux, O., \& Marquis, P. (1999). Distance-sat: Complexity and algorithms. In Proceedings of the Sixteenth National Conference on Artificial Intelligence (AAAI-99), pp. 642647.

Dechter, R. (1992). From local to global consistency. Artificial Intelligence, 55(1), 87-108.

Garey, M. R., \& Johnson, D. S. (1979). Computers and Intractability: A Guide to the Theory of NP-completeness. W. H. Freeman and Company, New York.

Ginsberg, M., Parkes, A., \& Roy, A. (1998). Supermodels and robustness. In Proceedings of the Fifteenth National Conference of the American Association for Artificial Intelligence and the tenth conference on Innovative Applications of Artificial Intelligence, 1998, Madison, WI, pp. 334-339.

Hebrard, E., Hnich, B., \& Walsh, T. (2004a). Robust solutions for constraint satisfaction and optimization. In Proceedings of the sixteenth European Conference on Artificial Intelligence, ECAI, pp. 186-190, Valencia, Spain.

Hebrard, E., Hnich, B., \& Walsh, T. (2004b). Super solutions in constraint programming. In Proceedings of the Internation Conference on Integration of AI and OR Techniques in Constraint Programming for Combinatorial Optimization Problems (CPAIOR), pp. $157-172$.

Holland, A., \& O'Sullivan, B. (2004). Super solutions for combinatorial auctions. In Proceedings of CSCLP 2004: Joint Annual Workshop of ERCIM/CoLogNet on Constraint Solving and Constraint Logic Programming.

Hoos, H., \& O’Neill, K. (2000). Stochastic local search methods for dynamic sat - an initial investigation. In AAAI-2000 Workshop Leveraging Probability and Uncertainty in Computation, pp. 22-26.

Jeavons, P. G., Cohen, D. A., \& Cooper, M. C. (1998). Constraints, consistency and closure. Artificial Intelligence, 101(1-2), 251-265.

Kautz, H. A., \& Selman, B. (1992). Planning as satisfiability. In Proceedings of the Tenth European Conference on Artificial Intelligence (ECAI'92), pp. 359-363.

Luks, E. M., \& Roy, A. (2005). Combinatorics of singly-repairable families. Electronic Journal of Combinatorics, 12(1), Research Paper 59, 17 pp. (electronic).

Papadimitriou, C. H. (1994). Computational Complexity. Addison-Wesley.

Schaefer, T. J. (1978). The complexity of satisfiability problems. In STOC 'r8: Proceedings of the tenth annual ACM symposium on Theory of computing, pp. 216-226. ACM Press. 